AperTO - Archivio Istituzionale Open Access dell'Università di Torino

\title{
Personalized therapeutic strategies in HER2-driven gastric cancer
}

\section{This is the author's manuscript}

Original Citation:

Availability:

This version is available http://hdl.handle.net/2318/1795119

since 2021-07-27T10:41:23Z

Published version:

DOI:10.1007/s10120-021-01165-w

Terms of use:

Open Access

Anyone can freely access the full text of works made available as "Open Access". Works made available under a Creative Commons license can be used according to the terms and conditions of said license. Use of all other works requires consent of the right holder (author or publisher) if not exempted from copyright protection by the applicable law. 
Original Article

\section{Personalized therapeutic strategies in HER2-driven gastric cancer}

Stefano Ughetto ${ }^{1,2 \S}$, Cristina Migliore ${ }^{1,2 \S}$, Filippo Pietrantonio ${ }^{3,4}$, Maria Apicella ${ }^{2}$, Annalisa Petrelli $^{2}$, Laura D’Errico ${ }^{1,2}$ Stefania Durando $^{2}$, Daniel Moya-Rull ${ }^{2}$, Sara E. Bellomo ${ }^{2}$, Sabrina Rizzolio $^{2}$, Tania Capelôa ${ }^{2 \#}$, Salvatore Ribisi², Maurizio Degiuli ${ }^{5}$, Rossella Reddavid ${ }^{5}$, Ida Rapa ${ }^{5}$, Uberto Fumagalli ${ }^{\ddagger}$, Stefano De Pascale ${ }^{6 \ddagger}$, Dario Ribero ${ }^{2}$, Carla Baronchelli ${ }^{7}$, Giovanni Sgroi ${ }^{8}$, Emanuele Rausa ${ }^{8}$, Gian Luca Baiocchi ${ }^{9}$, Sarah Molfino ${ }^{9}$, Stefania Manenti ${ }^{7}$, Maria Bencivenga ${ }^{10}$, Michele Sacco $^{10}$, Claudia Castelli ${ }^{10}$, Salvatore Siena ${ }^{4,11}$, Andrea Sartore-Bianchi ${ }^{4,11}$, Federica Tosi $^{4,11}$, Federica Morano ${ }^{3}$, Alessandra Raimondi ${ }^{3}$, Michele Prisciandaro ${ }^{3}$, Annunziata Gloghini ${ }^{12}$, Silvia Marsoni1 ${ }^{13 ¥}$, Antonino Sottile ${ }^{2}$, Ivana Sarotto ${ }^{2}$, Anna Sapino ${ }^{2,14}$, Caterina Marchiò ${ }^{2,14}$, Paola Cassoni $^{14}$, Simona Corso $^{1,2 *}$ and Silvia Giordano ${ }^{1,2 * \infty}$.

${ }^{1}$ Department of Oncology, University of Torino, Candiolo, Italy; ${ }^{2}$ Candiolo Cancer Institute, FPOIRCCS, Candiolo, Italy; ${ }^{3}$ Medical Oncology Department, Fondazione IRCCS Istituto Nazionale dei Tumori, Milan, Italy; ${ }^{4}$ Department of Oncology and Hemato-oncology, University of Milan, Milan, Italy; ${ }^{5}$ Department of Oncology, University of Torino, Orbassano, Italy; ${ }^{6}$ Chirurgia Generale 2, Spedali Civili, Brescia, Italy; ${ }^{7}$ Department of Pathology, ASST Spedali Civili, Brescia, Italy; ${ }^{8}$ Surgical Oncology Unit, Surgical Science Department, ASST Bergamo Ovest, Treviglio (BG), Italy; ${ }^{9}$ Department of Clinical and Experimental Sciences, Surgical Clinic, University of Brescia, Brescia, Italy; ${ }^{10}$ Department of Surgical Sciences, Dentistry, Gynecology and Pediatrics, Section of Surgery, University of Verona, Italy; ${ }^{11}$ Niguarda Cancer Center, Grande Ospedale Metropolitano Niguarda, Milan, Italy; ${ }^{12}$ Pathology and Laboratory Medicine Department, Fondazione IRCCS Istituto Nazionale dei Tumori, Milan, Italy; ${ }^{13}$ Istituto FIRC di Oncologia Molecolare (IFOM), Milan, Italy; ${ }^{14}$ Department of Medical Sciences, University of Torino, Italy;.

$\S$ These authors equally contributed to the work

* These authors equally contributed to the work

\#Current Affiliation: Pole of Pharmacology \& Therapeutics, Institut de Recherche Expérimentale et Clinique (IREC), Université catholique de Louvain (UCLouvain), Brussels, Belgium. 
$\$$ Current Affiliation: Chirurgia dell’Apparato Digerente - European Institute of Oncology IRCCS Milan, Italy.

${ }^{\ddagger}$ Current Affiliation: IFOM - The FIRC Institute of Molecular Oncology, Milan, Italy

Running Title: HER2 targeting in HER2 hyper-amplified gastric cancer

Keywords: HER2; targeted therapy; trastuzumab; gastric cancer; drug resistance

\section{Financial support}

This work was funded by the Italian Association for Cancer Research (AIRC), IG 20210 to SG, 21770 to SC, 23624 to FP and 22850 to CM; PRIN 2015 to SC; Fondazione Piemontese per la Ricerca sul Cancro (FPRC, ONLUS) 5X1000 Min. Salute 2013 to A.Sottile; FPRC 5X1000 2014 Min. Salute to SG and to A.Sapino; FPRC 5X1000 2015 Min. Salute to SG and to A. Sapino; FPRC 5X1000 2015 Min. Salute "Strategy" to SG; Ricerca Corrente 2019, Min. Salute to A.Sapino.

\section{Correspondence}

$\infty$ Silvia Giordano

Department of Oncology, University of Torino; Candiolo Cancer Institute, FPO-IRCCS, Strada Provinciale 142, Candiolo, 10060 (Torino), Italy. Phone: +39 011 9933233; Fax: +39 011 9933225; e-mail: silvia.giordano@unito.it

\section{Competing interests}

FP received honoraria from Amgen, Roche, Lilly, Bayer, Servier, Merck-Serono, Sanofi. Research grants from BMS; AS-B. participated in advisory boards for Amgen, Bayer, Samsung Bioepis and 
Sanofi. S. S is advisory board member for Amgen, Bayer, BMS, CheckmAb, Clovis, DaiichiSankyo, Merck, Roche-Genentech, and Seattle Genetics. CM has received personal/consultancy fees from Axiom Healthcare Strategies, Daiichi-Sankyo, MSD, Roche and Bayer.The remaining authors declare no potential conflicts of interest.

\section{Other notes}

Word count: 4027

Number of figures: 6

Supplementary Material: Supplementary methods; 6 Supplementary Figures; 1 Supplementary table; 


\begin{abstract}
Background: Trastuzumab is the only approved targeted therapy in patients with HER2 amplified metastatic gastric cancer. Regrettably, in clinical practice only a fraction of them achieves long term benefit from trastuzumab-based upfront strategy. To advance precision oncology, we investigated the therapeutic efficacy of different HER2-targeted strategies, in HER2 "hyper"-amplified (>8 copies) tumors.
\end{abstract}

Methods: We undertook a prospective evaluation of HER2 targeting with monoclonal antibodies, tyrosine kinase inhibitors and antibody-drug conjugates, in a selected subgroup of HER2 "hyper"amplified gastric patient-derived xenografts (PDXs), through the design of ad hoc preclinical trials. Results: Despite the high level of HER2 amplification, trastuzumab elicited a partial response only in 2 out of 7 PDX models. The dual HER2 blockade with trastuzumab plus either pertuzumab or lapatinib led to complete and durable responses in $5(71 \%)$ out of 7 models, including one tumor bearing a concomitant HER2 mutation. In a resistant PDX harboring KRAS amplification, the novel antibody-drug conjugate trastuzumab deruxtecan (but not trastuzumab emtansine) overcame KRAS-mediated resistance. We also identified a HGF-mediated non-cell-autonomous mechanism of secondary resistance to anti-HER2 drugs, responsive to MET co-targeting.

Conclusions: These preclinical randomized trials clearly indicate that in HER2-driven gastric tumors a boosted HER2 therapeutic blockade is required for optimal efficacy, leading to complete and durable responses in most of the cases. Our results suggest that a selected subpopulation of HER2-“hyper"-amplified GC patients could strongly benefit from this strategy. Despite the negative results of clinical trials, the dual blockade should be reconsidered for patients with clearly HER2addicted cancers.

Keywords: HER2; targeted therapy; trastuzumab; gastric cancer; drug resistance 


\section{INTRODUCTION}

Trastuzumab is the only approved targeted therapy in patients with metastatic gastric cancer (mGC) bearing overexpression/amplification of HER2, a molecular driver belonging to the Epidermal Growth Factor Receptor (EGFR) family of receptor tyrosine kinases (RTKs). Constitutive activation of the HER2 pathway, usually due to gene amplification or mutations, has been observed in several solid tumors where it drives tumor growth, metastasis and angiogenesis (1). HER2 is amplified in around $10-15 \%$ of GCs $(2,3)$ and is usually associated with the "chromosomal instability" (CIN) molecular subtype, although elevated intra-tumor and inter-lesion heterogeneity has been found in HER2-amplified GCs $(4,5)$. The post-hoc analysis of the ToGA trial demonstrated that the addition of the monoclonal antibody (MoAb) trastuzumab to chemotherapy significantly improved overall survival (OS) in patients with locally advanced or metastatic gastric or gastro-esophageal junction cancers, but exclusively in the subgroup with higher levels of HER2 expression (IHC $3+$ or IHC $2+$ with gene amplification). Regretfully, only a fraction of patients with HER2-amplified mGC clearly benefits from trastuzumab, casting doubts on the actual costeffectiveness of this regimen in clinical practice for the overall HER2-positive population. Indeed, the limitation of long-term efficacy of trastuzumab-based treatment may be due to the high percentage of primary and acquired resistance mechanisms involving RTK/KRAS coamplifications, PI3K/Akt axis deregulation, HER2 loss, heterogeneity of HER2 proteome including d16HER2 splice variants and, potentially, non-cell autonomous mechanisms (6-9).

Beyond trastuzumab, several HER2 targeted drugs are currently approved in patients with HER2positive breast cancer, including the MoAb pertuzumab combined with trastuzumab, the small molecule EGFR/HER2 tyrosine kinase inhibitor lapatinib and the antibody-drug conjugates (ADC) trastuzumab emtansine (T-DM1) and trastuzumab deruxtecam (DS-8201a). However, the phase 3 trials conducted with these agents in mGC were all negative (10-12). While in breast and gastric cancers trastuzumab is effective also as monotherapy, in other cancers -such as colorectal canceronly dual HER2 blockade with trastuzumab plus other anti-HER2 drugs (including pertuzumab or 
lapatinib) has shown significant activity (13-15). Notably, the level of HER2 amplification may greatly impact the long-term efficacy of trastuzumab, with HER2 gene copy number to chromosome 17 centromere ratio of 4.7 suggested as the optimal cut off value to identify patients with exceptional response (16). Based on the above assumptions, it is conceivable that the potential role of novel HER2-targeted strategies or dual anti-HER2 blockade should be re-assessed in molecularly selected patients with HER2 "hyper"-amplified/HER2 addicted cancers.

At present, the optimal preclinical model to validate targets and identify effective treatments is represented by Patient-Derived Xenografts (PDXs), which combine the versatility of preclinical evaluation with the informative significance of population-based studies $(17,18)$. Taking advantage of a proprietary, molecularly annotated colony of GC PDXs (19), we undertook a prospective evaluation of the therapeutic efficacy of different HER2 targeted strategies, in a selected subgroup of HER2 "hyper"-amplified xeno-patients through the design of ad hoc preclinical trials aimed at improving personalized treatment of HER2-positive GC. 


\section{MATERIALS AND METHODS}

\section{Patients}

Tumor samples (from gastric and gastroesophageal junction carcinomas) and matched normal samples were obtained from patients undergoing surgery in 15 Italian Hospitals (see Suppl. Methods). All patients provided written informed consent; samples were collected and the study was conducted under the approval of the Review Boards of all the Institutions. The study was done in accordance with the principles of the Declaration of Helsinki, the International Conference on Harmonization and Good Clinical Practice guidelines and GDPR (General Data Protection Regulation). Clinical and pathologic data were entered and maintained in our prospective database. All the samples have been anonymized before being shipped to Candiolo. No reference to the patients can be inferred from the histological and molecular characterization presented in the work.

\section{Primary cell cultures}

GC primary cells were derived from PDXs as described in(20). The genetic identity of the in vitroderived material with the original tumor has been verified by short tandem repeat profiling (Cell ID, Promega). For cell viability assays, cells were seeded in quadruplicate well in 96/well culture plates $\left(3-5 \times 10^{3}\right.$ cells/well), in the presence of the indicated drugs. After 6 days, cell viability was measured using the Cell TiterGlo Luminescent Cell Viability Assay (Promega).

\section{Western blot analysis}

Cells were treated for 24 hours with the indicated drugs, used at the concentration corresponding to IC50 in viability assays (as reported in figure legends). Whole-protein extracts were prepared using Laemmli buffer and quantified using the BCA Protein Assay kit (Pierce, Rockford, IL, USA).

\section{EBV evaluation}

Detection and quantification of EBV DNA were performed using the EBV Q-PCR Alert KIT (ELITechGroup S.p.A.). The real time amplification assay was carried out on ABI 7300 Real-Time PCR System instrument (Applied Biosystems, USA). PDXs were classified as described in(21): EBV high (with high EBV burden, >1000, Equivalent EBV Genomes/reaction (gEq), EBV 
intermediate $(75-1000 \mathrm{gEq})$ or EBV low/neg $(<75 \mathrm{gEq})$. Tumors scored as EBV high or intermediate were considered as EBV positive.

\section{PDX}

Generation. Gastric PDX generation was performed as described in(19). All animal procedures adhered to the 'Animal Research: Reporting of In Vivo Experiments' (ARRIVE) standards and were approved by the Ethical Committee of the Candiolo Cancer Institute (Candiolo, Torino, Italy), and by the Italian Ministry of Health.

Xenotrials. PDXs were passaged and expanded for $>2$ generations until production of a cohort of mice. Established and randomized tumors (average volume $250 \mathrm{~mm}^{3}$ ) were treated for the indicated days with the following regimens (either single agent or combination): vehicle (saline) per os; trastuzumab $30 \mathrm{mg} / \mathrm{Kg}$, weekly ip; pertuzumab $20 \mathrm{mg} / \mathrm{Kg}$, weekly ip; lapatinib $100 \mathrm{mg} / \mathrm{kg}$, daily, per os; TDM1 $10 \mathrm{mg} / \mathrm{Kg}$, weekly iv, DS-8201a $10 \mathrm{mg} / \mathrm{Kg}$, weekly iv; crizotinib $25 \mathrm{mg} / \mathrm{Kg}$, daily, per os. Tumor size was evaluated once-weekly by caliper measurements and approximate volume of the mass was calculated using the formula $4 / 3 \pi(D / 2)(d / 2)^{2}$, where $D$ is the major tumor axis and $d$ is the minor tumor axis.

Response to treatment. The response in mice has been evaluated using RECIST 1.1-like criteria, i.e. progressive disease $(\mathrm{PD}): \geq 35 \%$ increase from baseline; partial response $(\mathrm{PR}): \geq 50 \%$ reduction from baseline; stable disease (SD): intermediate variations from baseline(22). Statistical testing for pharmacological experiment was performed with GraphPAD PRISM Software 8.0, using Two-way ANOVA followed by Bonferroni multiple comparisons correction. Statistical significance: $n s=$ not significant; *p <0,05; **p <0,01; ***p <0,001; ****p <0,0001.

\section{Genomic sequencing}

DNA extracted from PDX models along with a sample of normal germline DNA from each patient were collected for next generation sequencing. Using standard methods, Illumina sequencing libraries were generated and subjected to hybrid capture with a focused targeted bait set of 243 
genes selected based on their alteration in prior studies of gastroesophageal cancer(19, 23). GTR0455 has been sequenced for Whole Exome on Illumina NovaSeq platform using the Agilent SureSelectXT Human All Exon V6 library (Macrogene Inc, Seoul, Korea).

\section{In situ Hybridization and Immunohistochemistry}

Dual-color FISH was performed on $4 \mu \mathrm{m}$ thick sections using probes for HER2 (17q12) and CEP17 (Vysis, Inc, Downers Grove, IL, USA), as previously described(24). IHC for HER2 was performed on $4 \mu \mathrm{m}$ thick sections in a centralized manner at Candiolo Cancer Institute, using the HercepTest ${ }^{\mathrm{TM}}$ per Dako Autostainer (Agilent). Immunohistochemistry for P-Met was performed using the P-MET (Tyr1234/1235) antibody AF2480 from R\&D Systems.

The RNAscope probe for mouse HGF (Mm-Hgf-O1 \#435381, Advaced Cell Diagnostics) was hybridized on $4 \mu \mathrm{m}$ FFPE slides following the RNAscope 2.5RED assay protocol (\#322452 and \#322360). Sequential slides were stained with a mouse-specific control probe (mmPPIB: Peptidylprolyl cis-trans isomerase B, not shown). After that, the same slides have been hybridized, through immunohistochemistry experiment, with anti-Human cytokeratin antibody (CloneAE1/AE3 \#M3515-DAKO Glostrup Denmark) following the standard protocol. To quantify the amount of positive signals in the stromal (pan-cytokeratin negative) areas, at least 2 digital images/slide have been captured at 20x magnification. RNAscope positive regions (red hue regions), were quantified using ImageJ Software (NIH). Briefly, nuclei enclosed into tumor areas (pan-cytocheratin staining), have been excluded from the analysis; the area corresponding to stromal compartment has been computed; the background has been subtracted. Color deconvolution was performed using HPAS as vector, in order to split in three images; one for each channel (first channel contains nuclei, second channel RNAscope positive dots). Finally, particles analysis was performed for the two channels by setting the same size and circularity for all the images in the same channel. Positivity for every analized images, as proxy of the amount of stromal HGF, was as follow: RNAscope positive area/(nuclei area + RNAscope positive area)*100. Analysis have been performed in blind. 


\section{RESULTS}

\section{Prevalence of HER2 amplification in gastric cancer PDXs and response to trastuzumab}

Preclinical and clinical data obtained from tumors displaying HER2 amplification have shown that the clinically relevant threshold is at least 8 gene copies (16). Thus, we analyzed by real time qPCR 570 primary GCs and identified primary tumors bearing $\geq 8$ HER2 gene copies (Suppl.Table 1). Eight of these patients were treated with a trastuzumab containing therapeutic regimen: among them six experienced response to treatment (SD or PR).

From the 570 samples we were able to generate PDXs in 151 cases. Among them, 7 PDXs were bearing $\geq 8$ gene copies (Suppl. Table 1). FISH analysis confirmed HER2 gene amplification and IHC analysis revealed that all the models were HER2 3+, with more than $90 \%$ of positive cells (Suppl. Fig. 1). PDX models showing a copy number gain $(\mathrm{CNG})$ of at least 8 gene copies were passaged in vivo until five tumor-bearing animals/treatment group were produced, in order to evaluate the effect of the pure HER2 inhibition, without the confounding effect of chemotherapy. When xenografts reached an average volume of $\sim 250 \mathrm{~mm}^{3}$, mice were treated with trastuzumab and tumor response was evaluated according to RECIST-like Criteria (see Methods and Figure Legend). As shown in Figure 1, only 4 out of the 7 models displayed a clinical response to trastuzumab, including two stable diseases (SD, GTR0277 and GTR0402) and two partial responses (PR, GTR0108 and GTR0233).

Trastuzumab plus lapatinib or pertuzumab combinatorial therapies are more effective than trastuzumab monotherapy in HER2 hyper-amplified GC PDXs.

To evaluate whether dual HER2 blockade may improve the efficacy in terms of response compared to trastuzumab monotherapy, we tested different HER2 targeted drugs or combinations in five HER2+ PDXs scoring 3+ at the IHC HercepTest and bearing >8 HER2 copies. These tumors, at least in principle, have the maximal probability of being trastuzumab sensitive (6). The different treatment groups were: (1) trastuzumab (“gold standard”); (2) pertuzumab (anti-HER2 MoAb 
mainly disrupting ligand-induced HER2 heterodimers); (3) lapatinib (dual HER2/EGFR TKI), (4) trastuzumab plus lapatinib; (5) trastuzumab plus pertuzumab; (6) vehicle. In order to evaluate the pure response to HER2 inhibition, mice did not receive any chemotherapy. As shown in Figure 2A, trastuzumab monotherapy led to 2 PR and 1 SD (GTR0108, GTR0233 and GTR0277 respectively); pertuzumab monotherapy had no therapeutic efficacy, while lapatinib achieved PR only in GTR0233 PDX. In 3 out of 5 cases (GTR0108, GTR0233, GTR0277, displaying 200, 50 and 300 HER2 gene copies, respectively), trastuzumab plus pertuzumab or lapatinib was significantly more effective than trastuzumab monotherapy, resulting in complete responses (CR) in 3 out of 3 cases. Interestingly, in the GTR0277 model, displaying around 300 HER2 gene copies, we identified a HER3 activating mutation (p.G284R) (25) that could be responsible for the relatively low sensitivity to trastuzumab monotherapy (achievement of SD, Suppl. Fig. 2A). Indeed, the dual HER2 block, interfering with heterodimers formation and activation, led to a complete response (Figure 2). From this PDX, we derived in vitro primary cells which maintained both $H E R 2$ amplification and the HER3 mutation (Suppl. Fig. 2B,C). In vitro experiments showed that combinatorial treatment with trastuzumab plus an anti-HER3 MoAb (MM-121/seribantumab) resulted in a strong growth inhibition (Suppl. Fig. 2D).

In 2 PDX models (GTR0108 and GTR0233), we performed long-term experiments to evaluate the possible onset of secondary resistance to the mono and combo treatments. As shown in Figure 2B, while resistance to trastuzumab monotherapy invariably emerged, we never observed tumor reappearance in animals treated with dual HER2 blockade combinations. Even more strikingly, in the combo-treated mice we did not observe tumor regrowth when we decided to stop the combos, meaning that the treatment could be regarded as curative. Notably the prolonged dual treatment did not result in any overt toxicity (not shown).

To investigate which pathways were inactivated by the different drugs/drug combinations, we performed biochemical studies on the available PDX-derived primary cells. GTR0233 and GTR0277 cells (in which HER2 amplification was confirmed by RT qPCR (Suppl. Fig. 2B and 3) 
were treated with trastuzumab and lapatinib, alone or in combination. Viability assays showed that also in vitro the combo treatment was significantly more effective than each drug used in monotherapy (Figure 3 A,B, left part). Western blot analysis showed that while monotherapy with either trastuzumab or lapatinib poorly affected activation of downstream transducers such as AKT, MAPK and S6 (evaluated as read out of the PI3K, RAS/MAPK and mTOR pathways respectively), the drug combination resulted in a strong inhibition of signal transduction (Figure 3A,B, right part). Very similar results were obtained with organoids derived from the GTR0108 PDX (Suppl. Fig. 4A-C). These in vitro findings strongly support the results obtained in the in vivo experiments where trastuzumab induced only SD or PR, while dual HER2 blockade combinations resulted in durable CRs.

In two cases (GTR0031 and GTR0109, displaying 10 and 8 HER2 copies, respectively), we did not observe any response to the investigated anti-HER2 strategies (Figure 2A). Genomic analysis of the GTR0031 model revealed the presence of KRAS co-amplification (8 copies), shown to be responsible for resistance to RTK targeting in different tumor contexts (8, 26-29) (Suppl. Fig. 5A). No putative genomic alteration likely sustaining trastuzumab resistance was identified in GTR0109. As the HER2-targeting ADC T-DM1, consisting of the humanized MoAb trastuzumab covalently linked to the cytotoxic agent DM1, is effective in breast cancer, we investigated whether T-DM1 could overcome trastuzumab resistance in these two non-responsive PDXs. As shown in Suppl. Fig. 5B, T-DM1 effectively inhibited GTR0109 (SD), but it was inactive in GTR0031. The new ADC trastuzumab deruxtecan (DS-8201a, consisting of trastuzumab covalently linked to the topoisomerase inhibitor deruxtecan) has recently shown clinical activity in patients with advanced breast cancer after failure of all standard anti-HER2 agents including T-DM1(30). As DS-8201a has shown preliminary activity also in patients with heavily pre-treated HER2 positive mGC (31), we administered this agent to GTR0031 PDXs. As displayed in Suppl. Fig. 5C, DS-8201a induced a CR in this PDX, refractory to trastuzumab, dual HER2 blockade and T-DM1. 


\section{PDX models recapitulate patients' response to Trastuzumab}

Only two PDXs (namely GTR0402 and GTR0455) of our GC platform derived from patients who received a trastuzumab containing therapy.

The patient originating GTR0402 PDX, after tumor removal, received first a chemo + trastuzumab regimen, leading to $\mathrm{PR}$, and later trastuzumab monotherapy as maintenance, resulting in a prolonged SD (Figure 4A depicts the clinical history of this patient). In the GTR0402 PDX model derived from the primary gastric adenocarcinoma (68 HER2 copies, Suppl. Table 1) we observed $\mathrm{SD}$ in response to trastuzumab (Figure 4B upper graph), similarly to what determined by trastuzumab monotherapy in the patient. In this PDX model we also evaluated whether (as observed in GTR0277, GTR0233 and GTR0108 models) the response could be improved by the addition of either lapatib or pertuzumab. Xenografts were thus randomized into 4 cohorts, and treated with (i) vehicle; (ii) trastuzumab; (iii) trastuzumab + lapatinib; (iv) trastuzumab+ pertuzumab. As reported in Figure 4B, the combos overperformed compared to trastuzumab monotherapy, leading to either PR (trastuzumab+pertuzumab) or CR (trastuzumab+lapatinib). From one lung metastasis resected at patient progression (Figure 4A), we could derive another PDX model (GTR0402_METS; 80 HER2 copies, Suppl. Table 1) that was expanded and randomized in the same cohorts as of the PDX derived from the primary tumor (Figure 4B, lower graph). Interestingly, PDXs derived from the metastatic tumor were not responsive to trastuzumab, mimicking again the patient's response. Even in this setting the two combos (trastuzumab + lapatinib and trastuzumab + pertuzumab) performed better than trastuzumab alone, inducing a temporary stabilization of disease. A much stronger response was induced by DS-8201a which led to tumor regression (Suppl. Fig. 5D), proving the activity of this drug conjugate also in the context of acquired resistance.

PDX0455 (80 HER2 gene copies, Suppl. Table 1) was derived from a biopsy of a tumor showing primary resistance to trastuzumab-containing treatment (progressive disease (PD) according to RECIST 1.1 criteria; Figure 5A). Genomic analysis of the primary tumor and of the derived GTR0455 PDX model revealed the presence of an activating HER2 mutation (p.S310Y (32)) at the 
allelic frequency of 95\% (Figure 5B). The PDX was serially passaged in mice until six tumorbearing animals were produced per experimental group. Xenografts were randomized into 4 cohorts, and treated with (i) vehicle; (ii) trastuzumab; (iii) lapatinib; (iv) trastuzumab plus lapatinib. In accordance to the clinical history of the donor patient, trastuzumab-treated GTR0455 mice were resistant to treatment and experienced disease progression (Figure 5C). No response was observed in lapatinib-treated mice but the combination trastuzumab plus lapatinib resulted in a strong reduction of tumor volume (Figure 5C, Suppl. Fig. 6). In vitro experiments performed in PDXderived cells (which maintained HER2 amplification and mutation, Suppl. Fig. 6B) showed poor susceptibility to either trastuzumab or lapatinib used as single-agents, but strong inhibition when used in combination (Suppl. Fig. 6C-D).

Overall our results show that the PDX models, in spite of the tumor heterogeneity, closely mirror the patient behaviour and thus represent an invaluable tool to test new therapeutic approaches.

\section{A non-cell autonomous mechanism sustains adaptive secondary resistance to HER2 inhibition.}

As already shown (Figure 2), prolonged treatment of the GTR0233 PDX with anti-HER2 compounds in monotherapy resulted in tumor relapse (Figure 2B and 6A). The genomic analysis of resistant tumors did not show any putative genomic alterations likely sustaining resistance to HER2 inhibition (data not shown). We thus investigated the onset of "adaptive" resistance sustained by activation of other receptor tyrosine kinases which could vicariate for HER2 activation. We have recently shown that TKIs can induce non-cell-autonomous adaptive resistance to MET and EGFR targeted therapies through the secretion by cancer-associated fibroblasts of the MET ligand, hepatocyte growth factor (HGF) (33). We thus wondered if this could be true also for HER2. Immunohistochemistry analyses showed increased phosphorylation of the MET receptor in lapatinib-resistant tumors compared to the matching sensitive ones (Figure 6B). In-situ hybridization with a mouse HGF RNA probe revealed that resistant tumors produced significantly 
more HGF than sensitive ones (Figure 6C,D). To prove that stromal HGF-induced MET activation does sustain resistance we performed an in vivo experiment co-treating resistant tumors -either few days after implant or when the tumors reached a volume of $250 \mathrm{~mm}^{3}$ - with both lapatinib and crizotinib (a dual MET/ALK inhibitor). As displayed in Figure 6E, we observed that dual MET/HER2 inhibition prevented and overcame resistance in the above-mentioned settings, respectively. These results identify $\mathrm{HGF}$ stromal production as a new mechanism sustaining acquired resistance to HER2 inhibition. 


\section{DISCUSSION}

Based on the results of the ToGA trial (3), the combination of chemotherapy with trastuzumab is considered the gold standard of treatment for patients with HER2-positive metastatic gastric cancer. However, less than $20 \%$ of patients clearly benefit from this treatment. In breast cancer, the double HER2 block provided by combining trastuzumab with pertuzumab has shown significantly better efficacy than trastuzumab monotherapy (34). The same strategy was assessed in HER2-positive advanced gastric cancer patients by the JACOB trial which compared first-line chemotherapy plus trastuzumab and pertuzumab with standard "ToGA" strategy. Even if median OS was nonsignificantly increased in the experimental arm, the formally negative results of the study reinforced the well-known questions about the real role of HER2 as a unique and dominant driver in GC (10). From this perspective, Gomez-Martin and colleagues showed that higher level of HER2 amplification significantly predicts increased benefit from trastuzumab-based therapy in patients with advanced GC (16). A mean HER2/CEP17 ratio of 4.7 was found as the optimal cutoff value identifying tumors where HER2 acts as a driver gene. Taking advantage of the unique opportunity provided by our wide platform of HER2 “hyper"-amplified GC PDXs, we compared the efficacy of trastuzumab monotherapy versus dual therapy (tratuzumab + pertuzumab or lapatinib) in this subpopulation of HER2-positive cancers theoretically responsive to trastuzumab. Our results show that despite the high level of HER2 amplification, trastuzumab elicited a PR only in 2 out of 7 PDXs, while dual therapy determined CR in 5 out of 7 cases (GTR0108; GTR0277; GTR0233; GTR0402; GTR0455). Most importantly, the deepness of response was significantly higher with the combos, leading to durable responses that in the two evaluated cases did not relapse even after drug withdrawal.

Thanks to in vitro studies performed in the available PDX-derived cells, we showed that while trastuzumab alone only slightly decreased the activation of HER2 and its downstream targets, dual therapy was able to strongly impair or even abrogate it. A genetic rational for the increased activity of trastuzumab + lapatinib or pertuzumab was found in one case, GTR0277, displaying an 
activating mutation in HER3 (p.G284R). It has been hypothesized that this HER3 mutant acquires an untethered conformation of the extracellular domain relative to WT and promotes oncogenic signaling in a HER2-dependent manner (25). Our results are in line with this hypothesis as the dual treatments were more active against HER2/HER3 heterodimers compared to trastuzumab alone and were as efficient as the dual HER2/HER3 MoAbs. As a matter of fact, the presence of HER3 activating mutations may be a candidate genomic predictor of resistance to trastuzumab monotherapy and its role should be clinically validated in the frame of randomized clinical trials such as JACOB.

All together these results suggest that the addition of either pertuzumab or lapatinib to trastuzumab may be more effective than trastuzumab alone in a subgroup of HER2 positive GC patients displaying high levels of HER2 amplification and in which HER2 may be regarded as the dominant driver of oncogene addiction. Not surprinsigly, also trastuzumab-based therapy ("ToGA strategy") may be more effective in patients with HER2 "hyper"-amplified tumors compared with the remaining subjects with HER2-positive disease and lower number of HER2 copies (35).

In a patient who showed primary resistance to trastuzumab-based treatment, we identified an activating HER2 mutation in the amplified HER2 gene (95\% of allelic frequence both in the primary tumor and in the PDX). Thanks to the matching PDX (GTR0455), we showed its resistance to trastuzumab or lapatinib monotherapies, but response to trastuzumab plus lapatinib combination. Also in this case, experiments performed in vitro in PDX-derived cells confirmed the poor efficacy of monotherapies compared to dual therapy. This result shows that cases with concomitant presence of specific activating HER2 mutations can be targeted more efficiently with dual therapy.

Two PDXs failed to respond to all anti-HER2 treatments. In these models we tested the activity of antibody-drug-conjugates already approved in breast cancer, such as trastuzumab-emantansine (TDM1, Kadcycla). This agent showed efficacy in one of the two models (GTR0109). Interestingly, in the resistant model (GTR0031) the new and more potent ADC trastuzumab deruxtecan (DS8201a) was higly active. Intriguingly, the latter PDX model is $K R A S$ co-amplificated (8 copies), 
which is a well-known biomarker of primary resistance to therapies targeting upstream receptors (8). While the highly promising activity of trastuzumab deruxtecan has been recently reported in a small cohort of patients with trastuzumab-resistant HER2-amplified GC (31), we provide here the biological rationale for the use of HER2-directed ADCs to efficiently treat also those tumors displaying either primary or acquired trastuzumab resistance.

Notably, in the only two cases where we could compare the response to trastuzumab in a patient and the corresponding PDX (namely GTR0402 and GTR0455) we observed a high similarity, further confirming the translational value of the obtained results.

Taken together, our results suggest that the role of dual HER2 blockade strategies should be reassessed by randomized clinical trials aimed at focusing the enrolment of patients with HER2positive GC to those with "hyper"-amplified status. Moreover, since the generation of evidencebased clinical data with novel targeted combinations is critically limited by the heterogeneity, multiplicity and dynamic evolution of resistance mechanisms to trastuzumab, as well as the undruggability of some of them (such as KRAS), the further clinical development of new ADCs such as trastuzumab deruxtecan is highly warranted and should proceed in parallel with pre-clinical platforms. 


\section{Authors' contributions}

SG and SC conceived and supervised the study and wrote the manuscript; SU, C.Migliore and FP contributed to design the experiments,; MD, RR, UF, SDP, CB, GS, ER, GLB, S.Molfino, MB, MS, CC, SS, ASB, FT, FM, AR, MP, DR provided patient material and data; C.Migliore, AP, LDE, SD, DMR, SEB, SU, SR, MA, TC, S. Ribisi performed experiments; SEB performed bioinformatics analyses; IS, PC, IR, AG, A.Sapino, C.Marchio' performed the pathologic analysis; A.Sottile provided technical support; S.Marsoni managed the GEA Study. All the authors revised the manuscript.

\section{Acknowledgements}

We thank Dr. Adam Bass for performing sequencing analysis of IRCC samples; our colleagues of GIRCG ('Gruppo Italiano Ricerca Carcinoma Gastrico’) for their support; M.C. Milanesio for data analysis; A. Balsamo for support in managing the GEA study; B.Martinoglio, M.Buscarino and M.Montone for technical support with Real Time PCR and Cell-ID; D.Balmativola, E.Maldi, M.Volante, A.Rigutto for pathological analysis; animal facility employees; L.Trusolino, A.Bertotti, C.Orru' and E.Puliga for helpful scientific discussion. SG and SC are EurOPDX Consortium members. 


\section{FIGURE LEGENDS}

Figure 1. Response to Trastuzumab treatment in PDXs bearing high HER2 CNG. The Spaghetti plot illustrates the effect of trastuzumab treatment $(30 \mathrm{mg} / \mathrm{kg})$ on PDXs with a HER2 $\mathrm{CNG} \geq 8$ copies (HER2 copies in each model are shown in brakets). Individual lines represent, for each PDX model, the mean percentage variation in tumor burden, from treatment start (day 0) to 4 weekly consecutive serial assessments ( $\mathrm{N}=5$ mice for each model). Tumor response has been evaluated using RECIST 1.1-like criteria according to (22): progressive disease (PD): $\geq 35 \%$ increase from baseline (pink background); partial response (PR): $\geq 50 \%$ reduction from baseline (green background); stable disease (SD): intermediate variations from baseline (yellow background).

\section{Figure 2. Dual HER2 blockade is the most effective and durable treatment in HER2-amplified}

PDXs. A. Tumor growth curves of mice cohorts derived from GTR0108, GTR0233, GTR0277, GTR0031 and GTR0109 patients, treated with the HER2 inhibitors trastuzumab, pertuzumab or lapatinib, alone or in combination, as indicated. Grey background: growth of the tumors before treatment start. The response in mice has been evaluated using RECIST 1.1-like criteria, progressive disease (PD): $\geq 35 \%$ increase from baseline (pink background); partial response (PR): $\geq$ $50 \%$ reduction from baseline (green background); stable disease (SD): intermediate variations from baseline (yellow background). Complete Response (CR): 100\% reduction from baseline. B. Tumor growth curves of mice cohorts derived from GTR0108 and GTR0233 patients undergoing prolonged ( $>6$ weeks) treatment with trastuzumab, or with the combos trastuzumab + lapatinib or trastuzumab + pertuzumab. Grey background: tumor growth before treatment start. The response in mice has been evaluated using RECIST 1.1-like criteria, as in A. The dashed line indicates stop of combo treatments. Mice receiving trastuzumab monotherapy continued the treatment until the end of the experiment or until mice were sacrificed for the tumor size. N=5 mice (GTR0108, GTR0233, GTR0277); N=6 mice (GTR0031; GTR0109); data are represented as mean + SD; *p <0,05; **p 
$<0,01 ; * * * \mathrm{p}<0,001 ; * * * * \mathrm{p}<0,0001$. Two-way ANOVA followed by Bonferroni multiple comparisons test has been used.

Figure 3. Dual HER2 blockade is more effective than trastuzumab alone in GTR0233 and GTR0277 PDX-derived cells in vitro. Cell viability assay performed on GTR0233 (A, left panel) and GTR0277 (B, left panel) tumor-derived cells, upon treatment for 6 days with the indicated

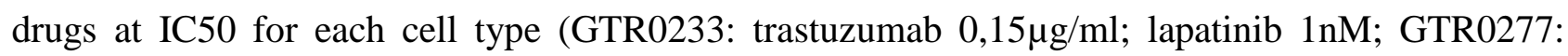
trastuzumab $10 \mu \mathrm{g} / \mathrm{ml}$; lapatinib $10 \mathrm{nM}$ ). Western blot analyses showing the activation state of HER2, EGFR and their downstream targets (AKT, MAPK and S6) in GTR0233 (A, right panel) and GTR0277 (B, right panel) tumor-derived cells treated for 24 hours with the indicated drugs/drug combinations (same doses used in the cell viability assays). Data are represented as mean of biological triplicates $+\mathrm{SD} ; * \mathrm{p}<0,05 ; * * \mathrm{p}<0,01 ; * * * \mathrm{p}<0,001$; ****p $<0,0001$. One-way ANOVA followed by Dunnett multiple comparisons test has been used

Figure 4. PDX models recapitulate patients' response to trastuzumab A. Summarized clinical course of the GTR0402 patient. Grey-lined boxes indicate periods of administration of the indicated therapeutic agents. Grey vertical lines indicate timing of tumor specimen acquisition from surgical procedures or biopsies, as well as dates of tumor assessment by CT scan. PD, progressive disease; PR, partial response; SD, stable disease (according to RECIST 1.1). The two red arrows indicate timing of specimen acquisition from which PDXs were derived. 5FU/LV: 5-fluorouracil/leucovorin; CDDP-5FU: Cisplatin, 5-fluorouracil. B. Tumor growth curves in mice cohorts derived from the GTR0402 tumor (upper graph) or from the GTR0402 metastasis (GTR0402_METS, lower graph), treated with vehicle, trastuzumab or the combos. Grey background: growth of the tumors before treatment start. The response in mice has been evaluated using RECIST 1.1-like criteria, as in figure 2. $\mathrm{N}=6$ mice; data are represented as mean $+\mathrm{SD}$; ****p $<0,0001$. Two-way ANOVA followed by Bonferroni multiple comparisons test has been used. 
Figure 5. The trastuzumab/lapatinib combo overcomes resistance to trastuzumab monotherapy in a HER2-mutated primary resistant PDX. A. Summarized clinical course of the GTR0455 patient. Grey-lined boxes indicate periods of administration of the indicated therapeutic agents. Grey vertical lines indicate timing of tumor specimen acquisition from surgical procedures or biopsies, as well as dates of tumor assessment by CT scan. PD, progressive disease; PR, partial response according to RECIST 1.1. The red arrow indicates timing of specimen acquisition from which PDX was derived. CDDP-5FU: Cisplatin, 5-fluorouracil; BSC: best supportive care. B. Detection of the HER2 S310Y mutation in the original tumor (Sanger sequencing, left panel) and in the GTR0455 PDX (exome sequencing, right panel). C. Tumor growth curves in mice cohorts derived from GTR0455 tumor, treated with vehicle, trastuzumab, lapatinib or the combo. Grey background: growth of the tumors before treatment start. The response in mice has been evaluated using RECIST 1.1-like criteria, as in figure 2. N=6 mice; data are represented as mean + SD; ****p $<0,0001$. Two-way ANOVA followed by Bonferroni multiple comparisons test has been used.

Figure 6. Identification of a non-cell autonomous, HGF-dependent mechanism of resistance to HER2 inhibition A. Generation of a lapatinib-resistant tumor. PDX GTR0233 has undergone prolonged treatment with lapatinib, until resistance onset. Grey background: growth of the tumors before treatment start. The response in mice has been evaluated using RECIST 1.1-like criteria, as in Figure 2. B. IHC (pMET staining) of tumor slices obtained from the vehicle-treated (upper panel) and the lapatinib-resistant tumor (lower panel). C. In situ hybridization with a murine-specific HGF probe (pink dots) of tumor slices obtained from the vehicle-treated (upper panel) and the lapatinibresistant tumor (lower panel). Slices have been also stained with panCytokeratin IHC to highlight tumor cells; D. Quantification of the mHGF signal in the stroma of tumors of either vehicle-treated or lapatinib-resistant tumors. E. Tumor growth curves of mice cohorts derived from the GTR0233 patient (LAP_resistant \#42), treated with the HER2 inhibitor lapatinib, alone or in combination with the MET inhibitor crizotinib, either few days after implant or when the tumors reached a volume of 
$250 \mathrm{~mm}^{3} . \mathrm{N}=5$ mice for each model; data are represented as mean $+\mathrm{SD}$; ***p <0,001; Two-way ANOVA followed by Bonferroni multiple comparisons test has been used. 


\section{REFERENCES}

1. Yarden $Y$, Sliwkowski MX. Untangling the ErbB signalling network. Nat Rev Mol Cell Biol. 2001;2(2):127-37. doi: 10.1038/35052073. PubMed PMID: 11252954.

2. Network CGAR. Comprehensive molecular characterization of gastric adenocarcinoma. Nature. 2014;513(7517):202-9. doi: 10.1038/nature13480. PubMed PMID: 25079317; PubMed Central PMCID: PMCPMC4170219.

3. Bang YJ, Van Cutsem E, Feyereislova A, Chung HC, Shen L, Sawaki A, et al. Trastuzumab in combination with chemotherapy versus chemotherapy alone for treatment of HER2-positive advanced gastric or gastro-oesophageal junction cancer (ToGA): a phase 3, open-label, randomised controlled trial. Lancet. 2010;376(9742):687-97. doi: 10.1016/S0140-6736(10)61121-X. PubMed PMID: 20728210.

4. Tajiri R, Ooi A, Fujimura T, Dobashi Y, Oyama T, Nakamura R, et al. Intratumoral heterogeneous amplification of ERBB2 and subclonal genetic diversity in gastric cancers revealed by multiple ligationdependent probe amplification and fluorescence in situ hybridization. Hum Pathol. 2014;45(4):725-34. Epub 2013/11/15. doi: 10.1016/j.humpath.2013.11.004. PubMed PMID: 24491355.

5. Park SR, Park YS, Ryu MH, Ryoo BY, Woo CG, Jung HY, et al. Extra-gain of HER2-positive cases through HER2 reassessment in primary and metastatic sites in advanced gastric cancer with initially HER2negative primary tumours: Results of GASTric cancer HER2 reassessment study 1 (GASTHER1). Eur J Cancer. 2016;53:42-50. Epub 2015/12/13. doi: 10.1016/j.ejca.2015.09.018. PubMed PMID: 26693898.

6. Browne BC, Crown J, Venkatesan N, Duffy MJ, Clynes M, Slamon D, et al. Inhibition of IGF1R activity enhances response to trastuzumab in HER-2-positive breast cancer cells. Ann Oncol. 2011;22(1):68-73. doi: 10.1093/annonc/mdq349. PubMed PMID: 20647220.

7. Berns K, Horlings HM, Hennessy BT, Madiredjo M, Hijmans EM, Beelen K, et al. A functional genetic approach identifies the PI3K pathway as a major determinant of trastuzumab resistance in breast cancer. Cancer Cell. 2007;12(4):395-402. doi: 10.1016/j.ccr.2007.08.030. PubMed PMID: 17936563.

8. Pietrantonio F, Fucà G, Morano F, Gloghini A, Corso S, Aprile G, et al. Biomarkers of Primary Resistance to Trastuzumab in HER2-Positive Metastatic Gastric Cancer Patients: the AMNESIA Case-Control Study. Clin Cancer Res. 2018;24(5):1082-9. Epub 2017/12/05. doi: 10.1158/1078-0432.CCR-17-2781. PubMed PMID: 29208673.

9. Jackson C, Browell D, Gautrey H, Tyson-Capper A. Clinical Significance of HER-2 Splice Variants in Breast Cancer Progression and Drug Resistance. Int J Cell Biol. 2013;2013:973584. Epub 2013/07/01. doi: 10.1155/2013/973584. PubMed PMID: 23935627; PubMed Central PMCID: PMCPMC3713377.

10. Tabernero J, Hoff PM, Shen L, Ohtsu A, Shah MA, Cheng K, et al. Pertuzumab plus trastuzumab and chemotherapy for HER2-positive metastatic gastric or gastro-oesophageal junction cancer (JACOB): final analysis of a double-blind, randomised, placebo-controlled phase 3 study. Lancet Oncol. 2018;19(10):137284. Epub 2018/09/11. doi: 10.1016/S1470-2045(18)30481-9. PubMed PMID: 30217672.

11. Thuss-Patience PC, Shah MA, Ohtsu A, Van Cutsem E, Ajani JA, Castro H, et al. Trastuzumab emtansine versus taxane use for previously treated HER2-positive locally advanced or metastatic gastric or gastro-oesophageal junction adenocarcinoma (GATSBY): an international randomised, open-label, adaptive, phase 2/3 study. Lancet Oncol. 2017;18(5):640-53. Epub 2017/03/23. doi: 10.1016/S1470-2045(17)301110. PubMed PMID: 28343975.

12. Hecht JR, Bang YJ, Qin SK, Chung HC, Xu JM, Park JO, et al. Lapatinib in Combination With Capecitabine Plus Oxaliplatin in Human Epidermal Growth Factor Receptor 2-Positive Advanced or Metastatic Gastric, Esophageal, or Gastroesophageal Adenocarcinoma: TRIO-013/LOGiC-A Randomized Phase III Trial. J Clin Oncol. 2016;34(5):443-51. doi: 10.1200/JCO.2015.62.6598. PubMed PMID: 26628478.

13. Sartore-Bianchi A, Trusolino L, Martino C, Bencardino K, Lonardi S, Bergamo F, et al. Dual-targeted therapy with trastuzumab and lapatinib in treatment-refractory, KRAS codon 12/13 wild-type, HER2positive metastatic colorectal cancer (HERACLES): a proof-of-concept, multicentre, open-label, phase 2 trial. Lancet Oncol. 2016. doi: 10.1016/S1470-2045(16)00150-9. PubMed PMID: 27108243.

14. Meric-Bernstam F, Hurwitz H, Raghav KPS, McWilliams RR, Fakih M, VanderWalde A, et al. Pertuzumab plus trastuzumab for HER2-amplified metastatic colorectal cancer (MyPathway): an updated 
report from a multicentre, open-label, phase 2a, multiple basket study. Lancet Oncol. 2019;20(4):518-30. Epub 2019/03/08. doi: 10.1016/S1470-2045(18)30904-5. PubMed PMID: 30857956; PubMed Central PMCID: PMCPMC6781620.

15. Strickler JH, Niedzwiecki D, Zemla T, Cercek A, Fakih M, $\mathrm{Ng} \mathrm{K}$, et al. A phase II, open label study of tucatinib (ONT-380) combined with trastuzumab in patients with HER2+ metastatic colorectal cancer (mCRC)(MOUNTAINEER). Journal of Clinical Oncology. 2017;35(15_suppl):TPS3624-TPS. doi: 10.1200/JCO.2017.35.15_suppl.TPS3624.

16. Gomez-Martin C, Plaza JC, Pazo-Cid R, Salud A, Pons F, Fonseca P, et al. Level of HER2 gene amplification predicts response and overall survival in HER2-positive advanced gastric cancer treated with trastuzumab. J Clin Oncol. 2013;31(35):4445-52. doi: 10.1200/JCO.2013.48.9070. PubMed PMID: 24127447. 17. Hidalgo $M$, Amant $F$, Biankin AV, Budinská E, Byrne AT, Caldas $C$, et al. Patient-derived xenograft models: an emerging platform for translational cancer research. Cancer Discov. 2014;4(9):998-1013. doi: 10.1158/2159-8290.CD-14-0001. PubMed PMID: 25185190; PubMed Central PMCID: PMCPMC4167608.

18. Byrne AT, Alférez DG, Amant F, Annibali D, Arribas J, Biankin AV, et al. Interrogating open issues in cancer precision medicine with patient-derived xenografts. Nat Rev Cancer. 2017. Epub 2017/01/20. doi: 10.1038/nrc.2016.140. PubMed PMID: 28104906.

19. Corso S, Isella C, Bellomo SE, Apicella M, Durando S, Migliore C, et al. A comprehensive PDX gastric cancer collection captures cancer cell intrinsic transcriptional MSI traits. Cancer Res. 2019. Epub 2019/10/04. doi: 10.1158/0008-5472.CAN-19-1166. PubMed PMID: 31585941.

20. Apicella M, Migliore C, Capelôa T, Menegon S, Cargnelutti M, Degiuli M, et al. Dual MET/EGFR therapy leads to complete response and resistance prevention in a MET-amplified gastroesophageal xenopatient cohort. Oncogene. 2017;36(9):1200-10. Epub 2016/08/15. doi: 10.1038/onc.2016.283. PubMed PMID: 27524418.

21. Corso S, Cargnelutti M, Durando S, Menegon S, Apicella M, Migliore C, et al. Rituximab Treatment Prevents Lymphoma Onset in Gastric Cancer Patient-Derived Xenografts. Neoplasia. 2018;20(5):443-55. Epub 2018/03/23. doi: 10.1016/j.neo.2018.02.003. PubMed PMID: 29574251; PubMed Central PMCID: PMCPMC5915970.

22. Bertotti A, Migliardi G, Galimi F, Sassi F, Torti D, Isella C, et al. A molecularly annotated platform of patient-derived xenografts ("xenopatients") identifies HER2 as an effective therapeutic target in cetuximabresistant colorectal cancer. Cancer Discov. 2011;1(6):508-23. doi: 10.1158/2159-8290.CD-11-0109. PubMed PMID: 22586653.

23. Pectasides E, Stachler MD, Derks S, Liu Y, Maron S, Islam M, et al. Genomic Heterogeneity as a Barrier to Precision Medicine in Gastroesophageal Adenocarcinoma. Cancer Discov. 2018;8(1):37-48. Epub 2017/10/04. doi: 10.1158/2159-8290.CD-17-0395. PubMed PMID: 28978556; PubMed Central PMCID: PMCPMC5894850.

24. Marchiò C, Dell'Orto P, Annaratone L, Geyer FC, Venesio T, Berrino E, et al. The Dilemma of HER2 Double-equivocal Breast Carcinomas: Genomic Profiling and Implications for Treatment. Am J Surg Pathol. 2018;42(9):1190-200. doi: 10.1097/PAS.0000000000001100. PubMed PMID: 29975246; PubMed Central PMCID: PMCPMC6110371.

25. Mishra R, Patel H, Alanazi S, Yuan L, Garrett JT. HER3 signaling and targeted therapy in cancer. Oncol Rev. 2018;12(1):355. Epub 2018/05/16. doi: 10.4081/oncol.2018.355. PubMed PMID: 30057690; PubMed Central PMCID: PMCPMC6047885.

26. Cargnelutti M, Corso S, Pergolizzi M, Mévellec L, Aisner DL, Dziadziuszko R, et al. Activation of RAS family members confers resistance to ROS1 targeting drugs. Oncotarget. 2015;6(7):5182-94. doi: 10.18632/oncotarget.3311. PubMed PMID: 25691052; PubMed Central PMCID: PMCPMC4467141.

27. Valtorta E, Misale S, Sartore-Bianchi A, Nagtegaal ID, Paraf F, Lauricella C, et al. KRAS gene amplification in colorectal cancer and impact on response to EGFR-targeted therapy. Int J Cancer. 2013;133(5):1259-65. doi: 10.1002/ijc.28106. PubMed PMID: 23404247.

28. Sasaki H, Yano M, Fujii Y. Evaluation of Kras gene mutation and copy number in thymic carcinomas and thymomas. J Thorac Oncol. 2010;5(10):1715-6. doi: 10.1097/JTO.0b013e3181f1cab3. PubMed PMID: 20871271. 
29. Sasaki H, Hikosaka Y, Kawano O, Moriyama S, Yano M, Fujii Y. Evaluation of Kras gene mutation and copy number gain in non-small cell lung cancer. J Thorac Oncol. 2011;6(1):15-20. doi: 10.1097/JTO.0b013e31820594f0. PubMed PMID: 21150464.

30. Modi S, Saura C, Yamashita T, Park YH, Kim SB, Tamura K, et al. Trastuzumab Deruxtecan in Previously Treated HER2-Positive Breast Cancer. N Engl J Med. 2020;382(7):610-21. Epub 2019/12/11. doi: 10.1056/NEJMoa1914510. PubMed PMID: 31825192.

31. Shitara K, Iwata H, Takahashi S, Tamura K, Park H, Modi S, et al. Trastuzumab deruxtecan (DS8201a) in patients with advanced HER2-positive gastric cancer: a dose-expansion, phase 1 study. Lancet Oncol. 2019;20(6):827-36. Epub 2019/04/29. doi: 10.1016/S1470-2045(19)30088-9. PubMed PMID: 31047804.

32. Kavuri SM, Jain N, Galimi F, Cottino F, Leto SM, Migliardi G, et al. HER2 activating mutations are targets for colorectal cancer treatment. Cancer Discov. 2015;5(8):832-41. doi: 10.1158/2159-8290.CD-141211. PubMed PMID: 26243863; PubMed Central PMCID: PMCPMC4527087.

33. Apicella M, Giannoni E, Fiore S, Ferrari KJ, Fernández-Pérez D, Isella $C$, et al. Increased Lactate Secretion by Cancer Cells Sustains Non-cell-autonomous Adaptive Resistance to MET and EGFR Targeted Therapies. Cell Metab. 2018;28(6):848-65.e6. Epub 2018/08/30. doi: 10.1016/j.cmet.2018.08.006. PubMed PMID: 30174307.

34. Swain SM, Baselga J, Kim SB, Ro J, Semiglazov V, Campone M, et al. Pertuzumab, trastuzumab, and docetaxel in HER2-positive metastatic breast cancer. N Engl J Med. 2015;372(8):724-34. doi: 10.1056/NEJMoa1413513. PubMed PMID: 25693012; PubMed Central PMCID: PMCPMC5584549.

35. Janjigian YY, Sanchez-Vega F, Jonsson P, Chatila WK, Hechtman JF, Ku GY, et al. Genetic Predictors of Response to Systemic Therapy in Esophagogastric Cancer. Cancer Discov. 2018;8(1):49-58. Epub 2017/11/09. doi: 10.1158/2159-8290.CD-17-0787. PubMed PMID: 29122777; PubMed Central PMCID: PMCPMC5813492. 
Figure 1

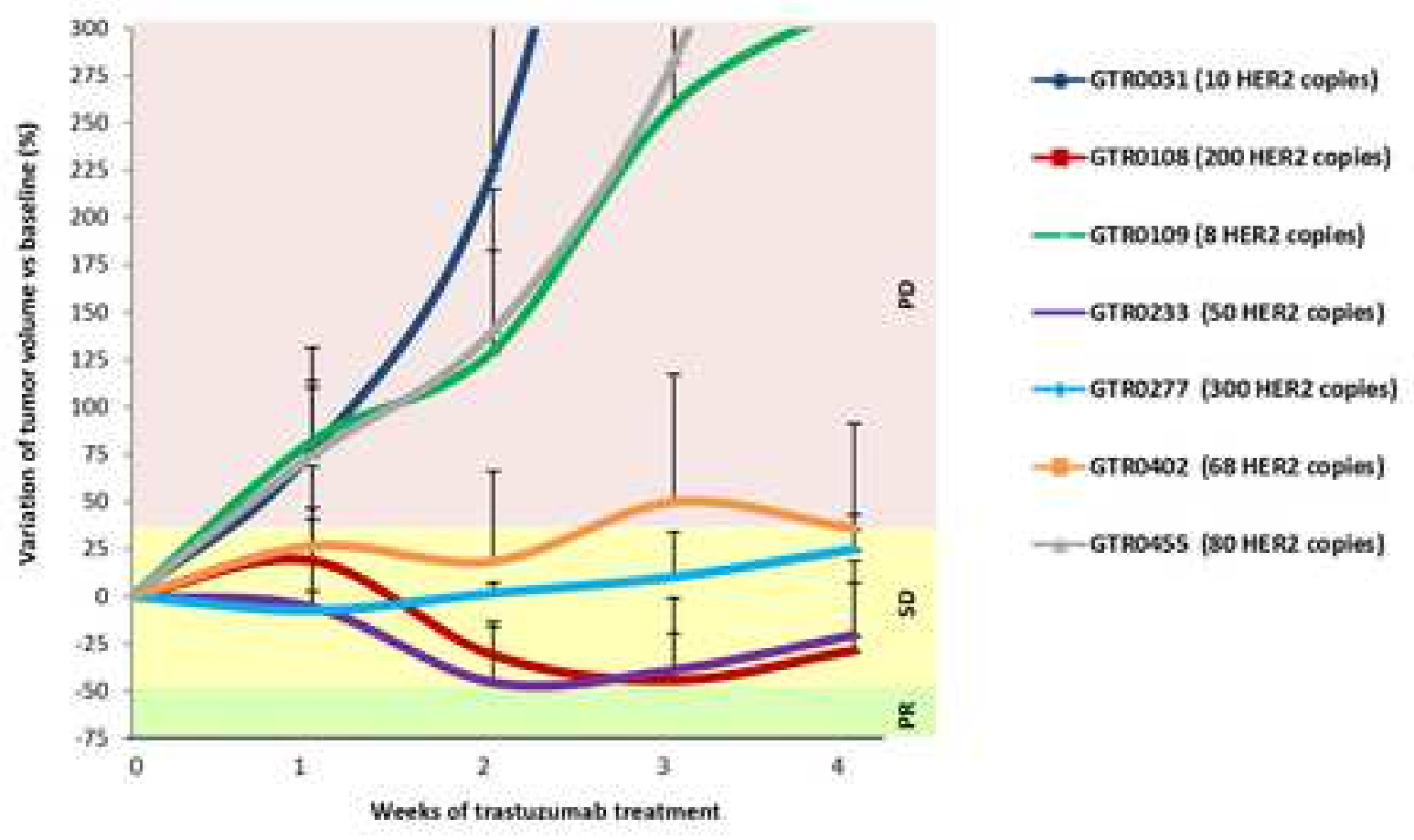


Figure 2
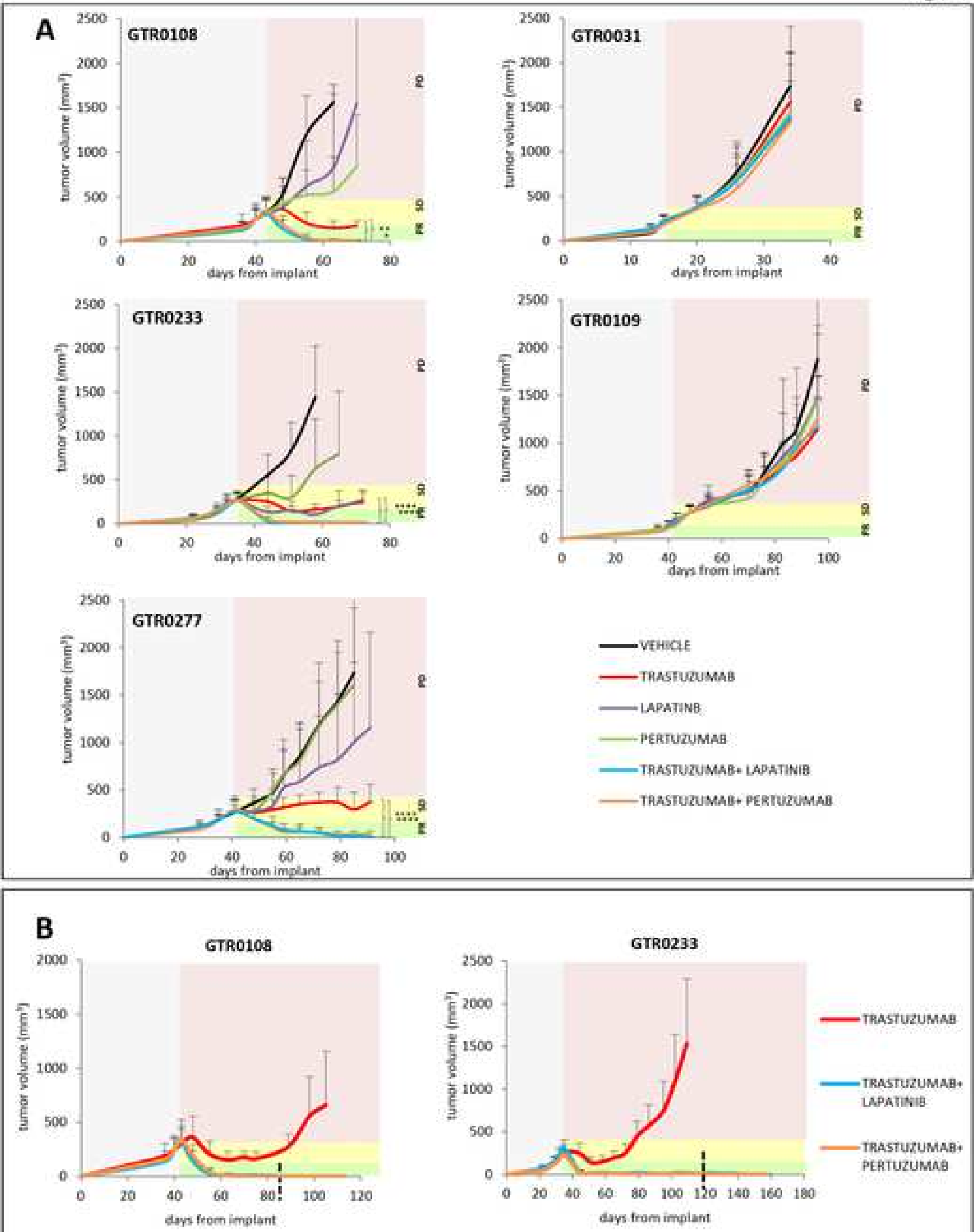
B
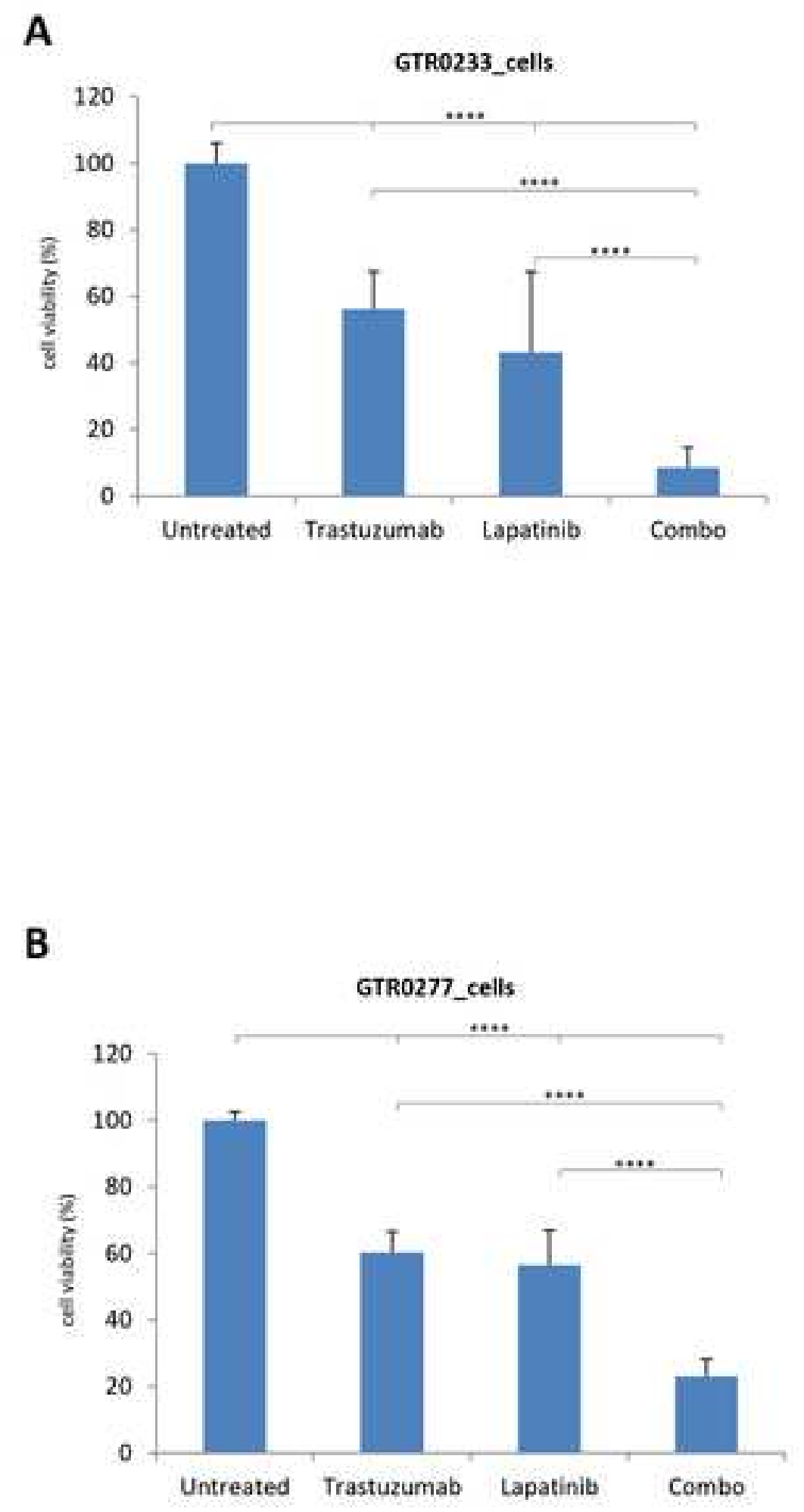
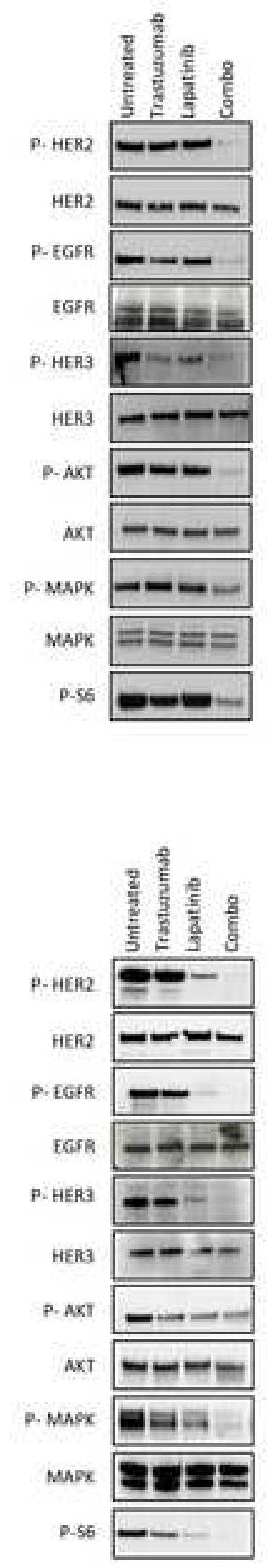

Figure 3 
Figure 4

\section{A}

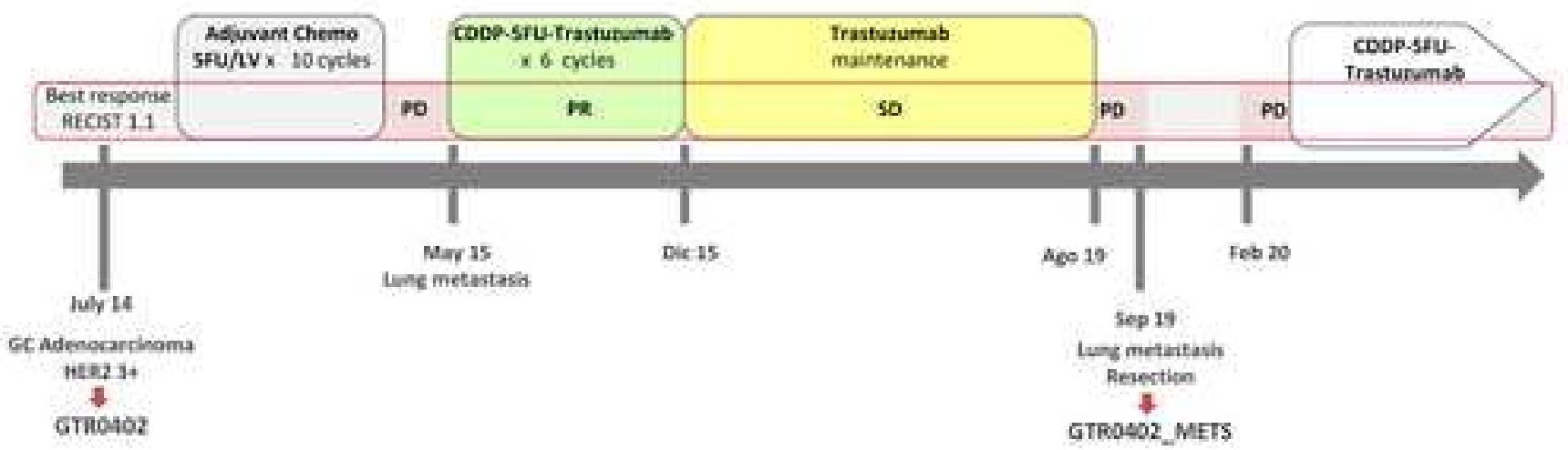

B
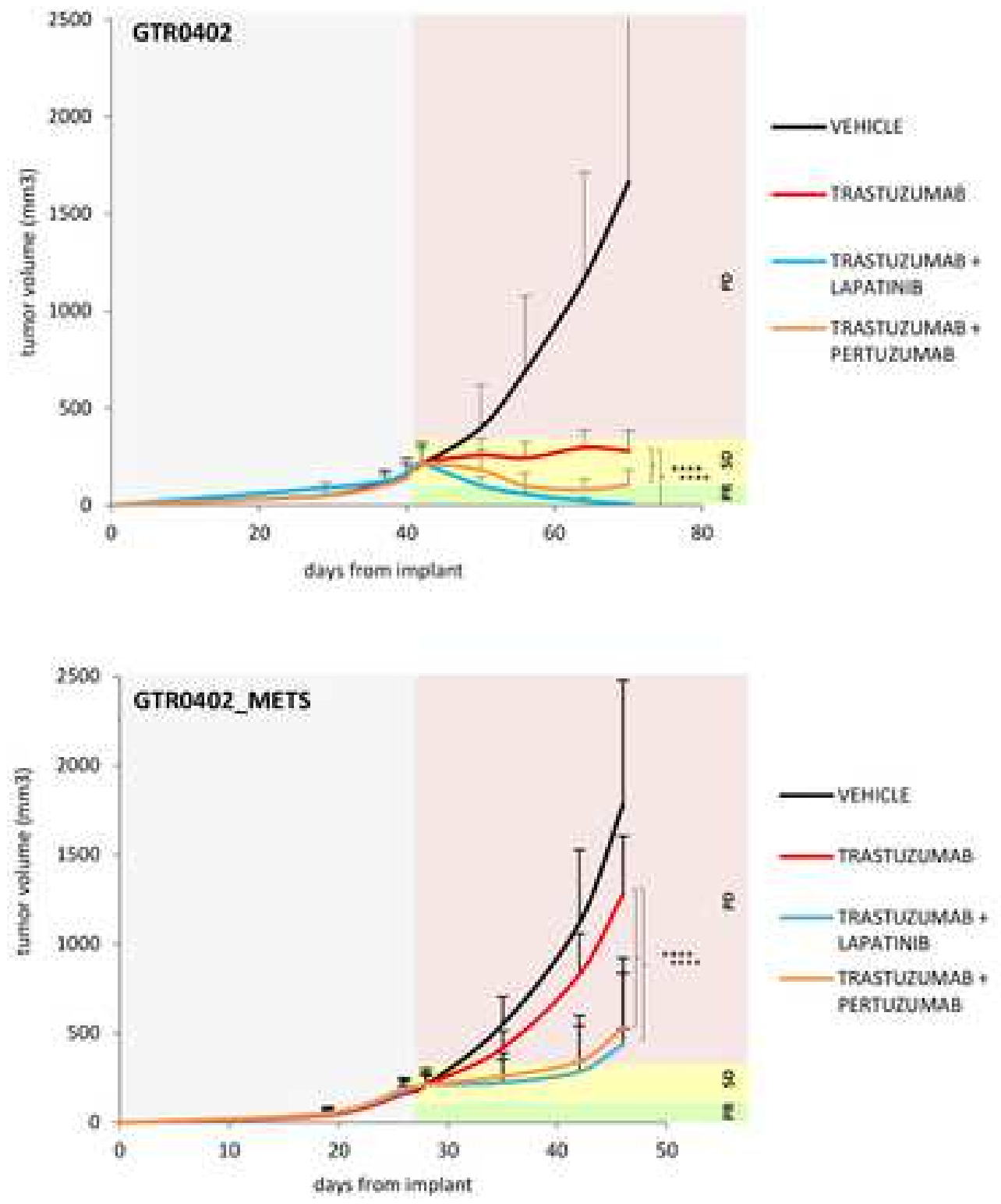
Figure 5
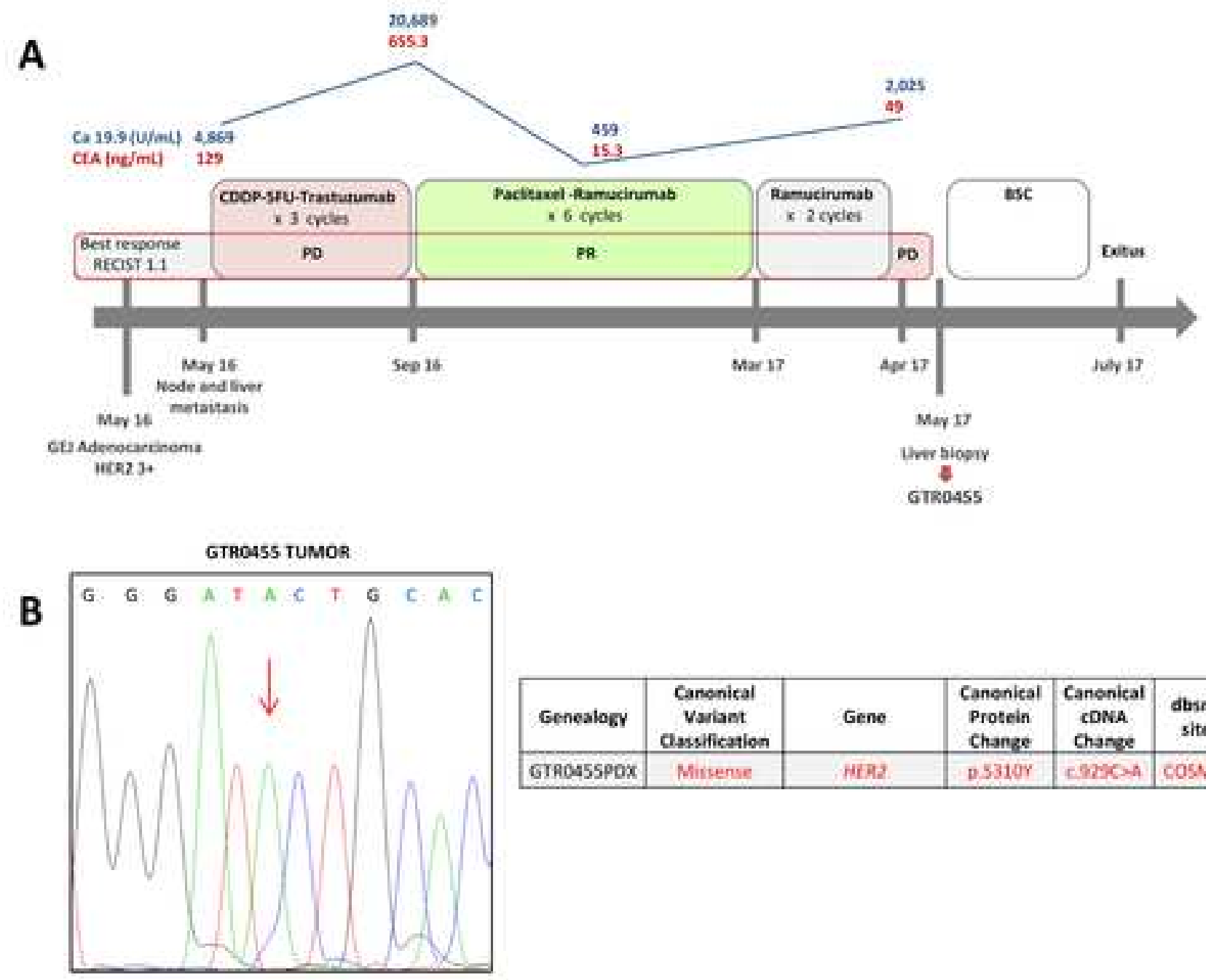

\begin{tabular}{|c|c|c|c|c|c|c|}
\hline Genealogr & $\begin{array}{c}\text { Canonical } \\
\text { Variant } \\
\text { Cassification }\end{array}$ & Gene. & $\begin{array}{l}\text { Canenital } \\
\text { Protein } \\
\text { Change }\end{array}$ & $\begin{array}{l}\text { Canonical } \\
\text { cDNA } \\
\text { Change }\end{array}$ & $\begin{array}{l}\text { dbsap } \\
\text { site }\end{array}$ & $\begin{array}{c}\text { Allele } \\
\text { fraction }\end{array}$ \\
\hline GrRos5spox & Mirsense & HED2 & p\$310r & $-97 \times-4$ & $\cos 2 x$ & 0.952 \\
\hline
\end{tabular}

\section{C}

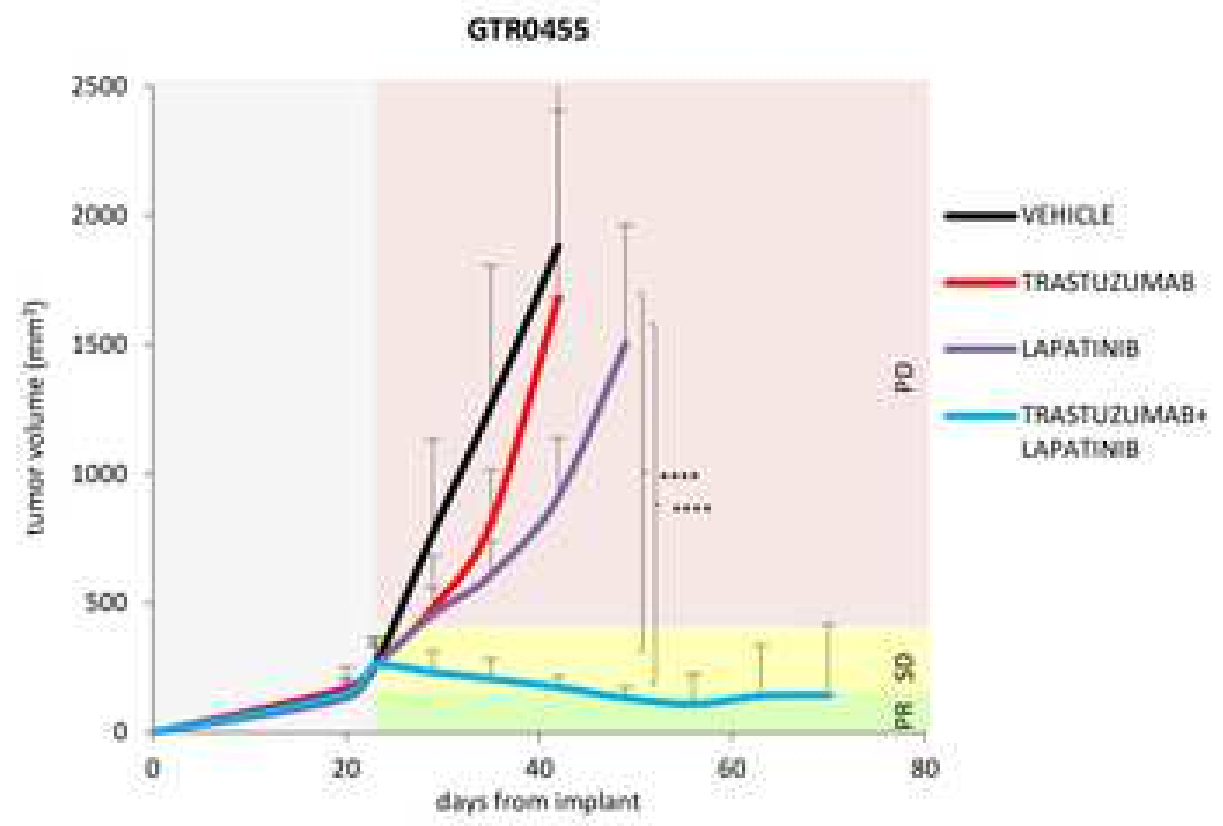


A

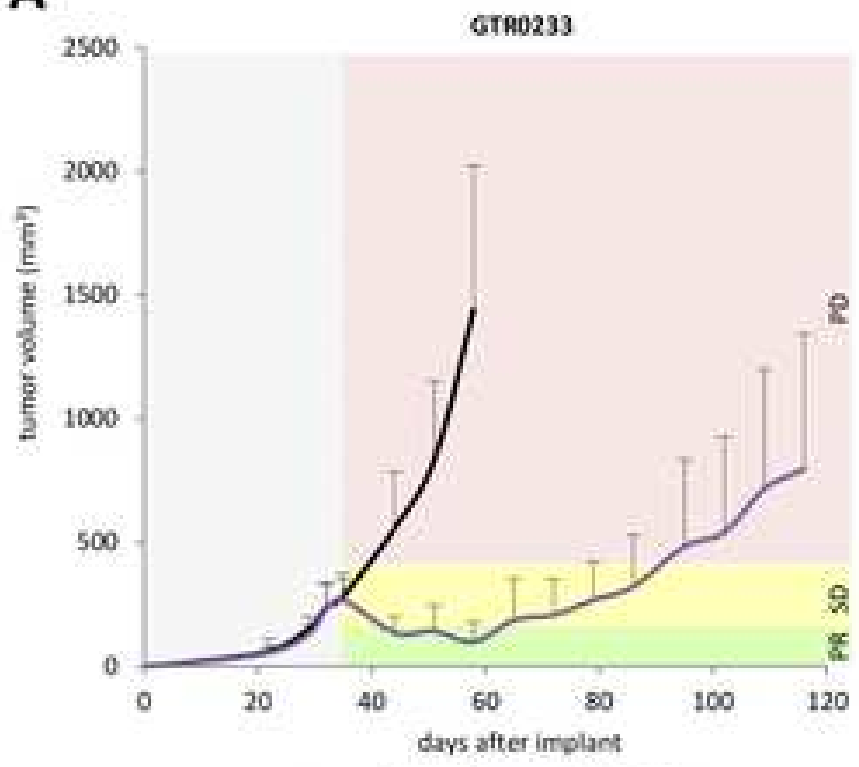

— VEHICLE - LAPATING

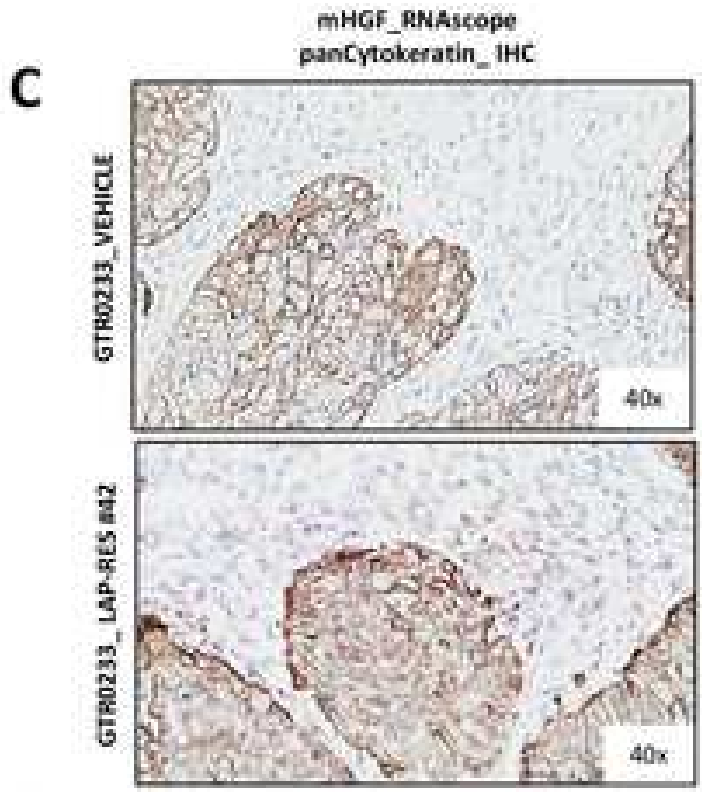

E

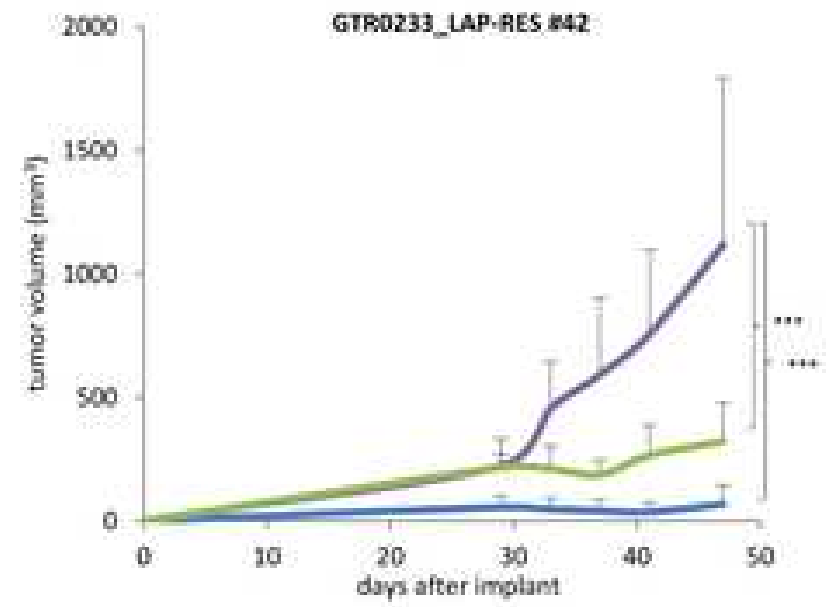

B
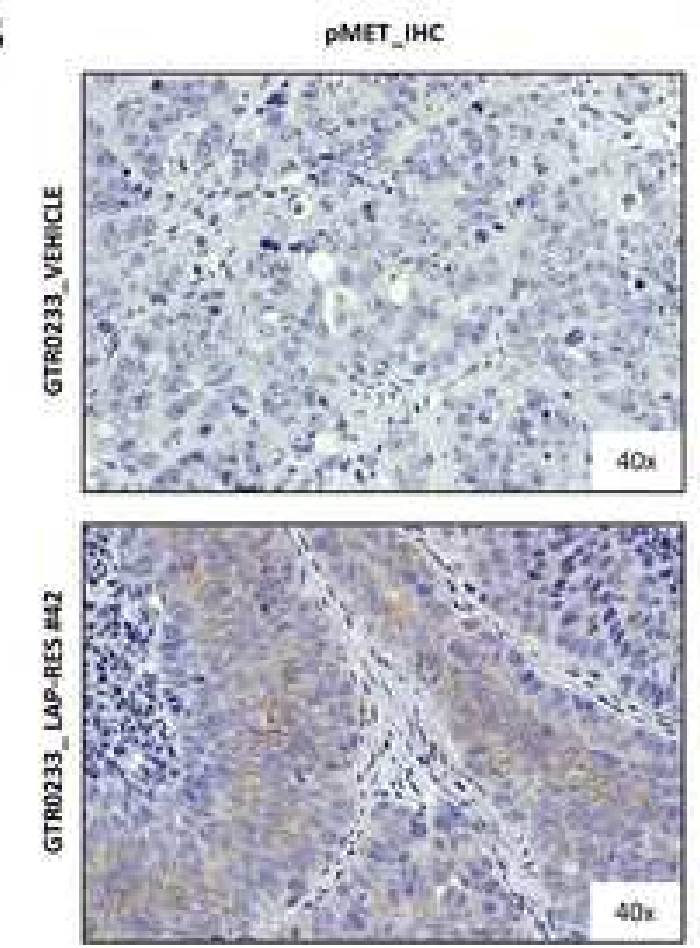

D

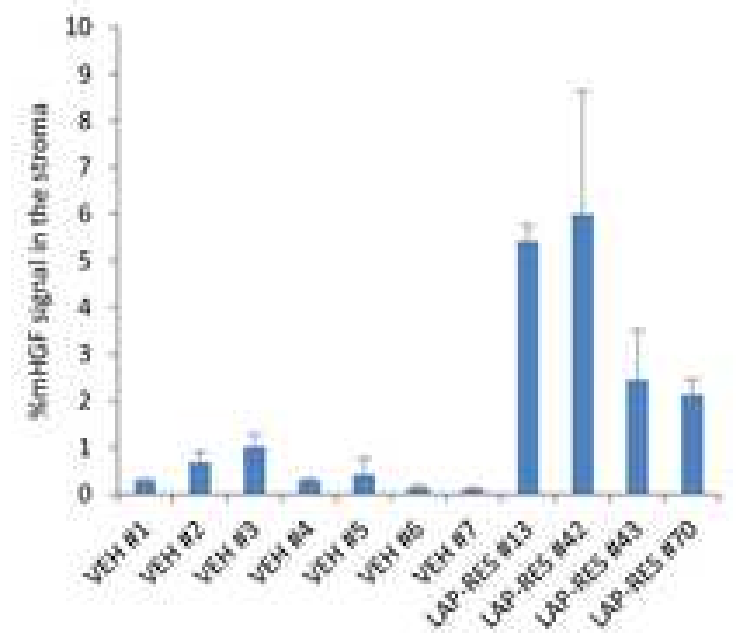

Figure 6

-LAPATINIB

- LAPATINHE. CRIZOTINIB

LAPATINIB. CRUzOTINIE tram day 30 
Mini-abstract (less than 30 words)

\begin{abstract}
Preclinical trials performed in selected HER2-“hyper"-amplified GC PDXs advice that a boosted
HER2 blockade should be reevaluated for patients in which HER2 may be regarded as the dominant driver of oncogene addiction.
\end{abstract}

\title{
.
} domitant driver of oncogene addiction.

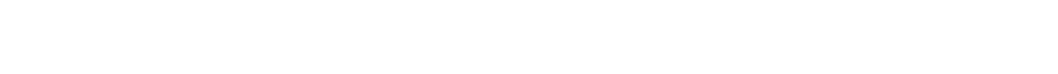

\section{MINI-ABSTRACT:}



Click here to access/download Electronic Supplementary Material SUPPLEMENTARY METHODS.docx 
Click here to access/download Electronic Supplementary Material Ughetto et al._Supplementary Table 1.docx 
Click here to access/download Electronic Supplementary Material Ughetto et al._Suppl. Fig.1.pdf 
Click here to access/download Electronic Supplementary Material Ughetto et al._Suppl. Fig.2.pdf 
Click here to access/download Electronic Supplementary Material Ughetto et al._Suppl. Fig.3.pdf 
Click here to access/download Electronic Supplementary Material Ughetto et al._Suppl. Fig.4.pdf 
Click here to access/download Electronic Supplementary Material Ughetto et al._Suppl. Fig.5.pdf 
Click here to access/download Electronic Supplementary Material Ughetto et al._Suppl. Fig.6.pdf 